\title{
Hsp90 inhibition results in autophagy-mediated proteasome-independent degradation of IKB kinase (IKK)
}

\author{
Guoliang Qing ${ }^{1}$, Pengrong Yan $^{1}$, Gutian Xiao ${ }^{1}$ \\ ${ }^{1}$ Department of Cell Biology and Neuroscience, Rutgers, The State University of New Jersey, 604 Allison Road, Piscataway, NJ \\ 08854, USA
}

Autophagic and proteasomal proteolysis are two major pathways for degradation of cellular constituents. Current models suggest that autophagy is responsible for the nonselective bulk degradation of long-lived proteins and organelles while the proteasome specifically degrades short-lived proteins including misfolded proteins caused by the absence of

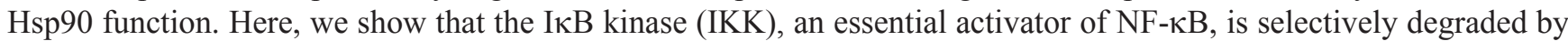
autophagy when Hsp90 is inhibited by geldanamycin (GA), a specific Hsp90 inhibitor showing highly effective anti-tumor activity. We find that in this case inactivation of ubiquitination or proteasome fails to block IKK degradation. However, inhibition of autophagy by an autophagy inhibitor or knockout of Atg5, a key component of the autophagy pathway, significantly rescues IKK from GA-induced degradation. These findings provide the first evidence that an Hsp90 client may be degraded by a mechanism different from the proteasome pathway and establish a molecular link among Hsp90, NF- $\kappa \mathrm{B}$ and autophagy

Cell Research (2006) 16:895-901. doi:10.1038/sj.cr.7310109; published online 7 November 2006

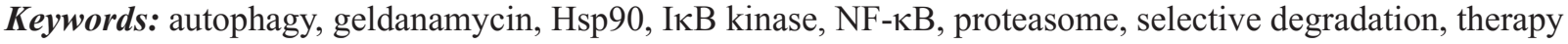

\section{Introduction}

Cellular homeostasis requires a well-controlled balance between protein synthesis and degradation. While protein degradation is mediated primarily by the ubiquitin-proteasome and autophagy-lysosome pathways, protein synthesis involves a series of processes, including mRNA transcription, protein translation, protein folding/maturation and subsequent conformation maintenance $[1,2]$. The correct folding and stability of a number of signaling molecules, including many kinases and transcription factors, requires the molecular chaperone Hsp90 (heat-shock protein of $90 \mathrm{kDa})$ and its co-chaperones [3, 4]. Specific inhibition

\footnotetext{
Correspondence: Gutian Xiao

Tel.: +1-732-445-2839; Fax: +1-732-445-5870;

E-mail:xiao@biology.rutgers.edu

Abbreviations: AICAR, 5-aminoimidazole-4-carboxamide 1-b-D-ribofuranoside; CMA, chaperone-mediated autophagy; GA, geldanamycin; Hsc70, heat-shock cognate of $70 \mathrm{kDa}$; Hsp90, heat-shock protein of $90 \mathrm{kDa}$; IкB, inhibitor of NF- $\kappa B$; IKK, IкB kinase

Received 28 September 2006; revised 9 October 2006; accepted 10 October 2006; published online 7 November 2006
}

of Hsp90 chaperone function by geldanamycin (GA), an anti-tumor drug, leads to degradation of its clients $[4,5]$. So far, the degradation of all known Hsp90 clients induced by $\mathrm{GA}$ is mediated by the proteasome $[3,4]$. Yet, to date, it has not been established whether the autophagy-lysosome pathway plays a role in GA-induced degradation of Hsp90 clients.

In mammals, three modes of autophagy have been identified: macroautophagy, microautophagy and chaperone-mediated autophagy (CMA). These three modes differ with respect to the pathway by which cytoplasmic material is delivered to the lysosome, but share in common the final steps of lysosomal degradation of the cargo with eventual recycling of the degraded material $[1,6]$. In CMA, the substrate protein is specifically recognized by a chaperone complex containing Hsc 70 (heat-shock cognate of $70 \mathrm{kDa}$ ) and then delivered into the lysosome. In microautophagy, the cargo is engulfed directly at the lysosomal surface by invagination, protusion and/or septation of the lysosomal membrane. In contrast, macroautophagy is characterized by the fact that the cargo is sequestered into a double membrane structure termed autophagosome before deliv- 
ery to the lysosome. Macroautophagy is the main form of autophagy and usually referred to directly as autophagy (same herein).

Recently, the IкB kinase (IKK) has been found to be a novel client of Hsp90 [7]. IKK is a protein complex composed of three subunits, IKK $\alpha$ (IKK1), IKK $\beta$ (IKK2) and IKK $\gamma(\mathrm{NEMO})$, which directly phosphorylates I $\mathrm{KBs}$ (inhibitors of NF- $\kappa \mathrm{B}$ ) for subsequent proteasomal degradation. The degradation of I $\kappa B$ s leads to activation of NF- $\kappa \mathrm{B}$, a transcription factor family involved in diverse biological processes $[8,9]$. Here, we demonstrate that all three subunits of IKK are selectively degraded by autophagy when Hsp90 is inhibited by GA. We find that inactivation of ubiquitination or proteasome fails to block IKK degradation induced by GA. However, biochemical or genetic inhibition of the autophagic pathway significantly rescues IKK from GA-induced degradation.

\section{Materials and Methods}

\section{Expression vectors and reagents}

Expression vectors encoding IKK have been described as before [10]. The anti-Hsp90 (F-8) and anti-p53 (FL-393) antibodies were from Santa Cruz Biotech Inc. The anti-actin antibody (AAN01) was from Cytoskeleton Inc. Other antibodies were described previously [11-14]. MG132 and 5-aminoimidazole-4-carboxamide 1- $b$-D-ribofuranoside (AICAR) were from Calbiochem and Biomol, respectively.

\section{Cell culture and transfection}

Human B-cell line Ramos RG69, mouse fibroblasts ts20 and Atg5 knockout mouse embryonic fibroblasts (MEFs) were gifts from Drs Covey L, Ozer HL and Mizushima N, respectively. Human kidney 293 cells and Jurkat cells were described previously [15]. 293, ts20 and MEF cells were cultured in Dulbecco's modified Eagle's medium supplemented with $10 \%$ fetal bovine serum and $2 \mathrm{mM} \mathrm{L}$-glutamine. Ramos RG69 cells and Jurkat cells were cultured in RPMI supplemented with $10 \%$ fetal bovine serum and $2 \mathrm{mM}$ L-glutamine. ts 20 cells were usually maintained at $35^{\circ} \mathrm{C}$ instead of $37^{\circ} \mathrm{C}$. For inactivation of E1 in the ts 20 cells, the culture temperature was shifted to 39 ${ }^{\circ}$ C. 293, ts20 and MEF cells were transfected with DEAE-Dextran and LipofectAMINE 2000 (Invitrogen), respectively [16, 17].

\section{Immunoblotting}

Cells were lysed in radioimmuoprecipitation assay buffer (RIPA buffer) (50 mM Tris-HCl pH 7.4, $150 \mathrm{mM} \mathrm{NaCl}, 1 \mathrm{mM}$ EDTA, $0.25 \%$ Na-deoxycholate, $1 \%$ NP-40, $1 \mathrm{mM}$ dithiothreitol and $1 \mathrm{mM}$ phenylmethylsulfony fluoride). About $30 \mu \mathrm{g}$ whole-cell lysates were fractionated by SDS-PAGE, transferred to nitrocellulose membranes and subjected to immunoblotting (IB) using the indicated antibodies $[11,15]$. To detect MG132 recovery of Akt from GA-mediated degradation by IB assay, the whole-cell lysates were prepared with the RIPA buffer containing 1\% SDS [18].

\section{Polysome and RNA isolation}

Ramos RG69 cells were treated for $2 \mathrm{~h}$ with anti-CD40 antibody $(10 \mu \mathrm{g} / \mathrm{ml})$ or GA $(2 \mu \mathrm{M})$ or left untreated. $20 \%$ of treated or untreated cells $\left(2 \times 10^{7}\right.$ cells/each group) were pelleted and employed as a source for total RNA using Trizol reagent (Invitrogen). The remaining cells were incubated with cycloheximide $(100 \mu \mathrm{g} / \mathrm{ml})$ for $15 \mathrm{~min}$, followed by cytoplasm extraction. The cytoplasmic extract was then loaded onto a linear $10-45 \%(\mathrm{w} / \mathrm{w})$ sucrose gradient and centrifuged for $2 \mathrm{~h} 30 \mathrm{~min}$ at $36000 \mathrm{rpm}$. with a Beckman SW-41 rotor. After centrifugation, the gradient was fractionated and its absorbance at $254 \mathrm{~nm}$ was determined continuously by an Isco UA-5 monitor as described before [13]. The polysome-containing fractions were pooled and subjected to phenol extraction. RNA was precipitated with ethanol and dissolved in DEPC-treated water.

\section{Real-time reverse transcription- $P C R$ analysis}

Two micrograms of total RNA or RNA isolated from polysomes were reverse-transcribed for real-time reverse transcription (RT)-PCR using the following primers:

human IKK $\alpha$ : forward 5'-CCA CTA TGC TGA GGT TGG TGT, reverse 5'-AGT CTC CCT GAC GTC TTC CAT;

human IKK $\beta$ : forward 5'-TAG CAT GAA TGC CTC TCG ACT, reverse 5'-TTC AGC CAC CAG TTC TTC ACT;

human IKK $\gamma$ : forward 5'-TAT CTA CAA GGC GGA CTT CCA, reverse 5'-TGG CCT TCA GTT TGC TGT ACT;

human p100: forward 5'-TGC CAT TGT GTT CCG GAC A, reverse 5'-TGT TTG GAA TCA GAC ACG TCC C;

human GAPDH: forward 5'-GCA AAT TCC ATG GCA CCG T, reverse 5'-TCG CCC CAC TTG ATT TTG G.

Real-time PCR assays were performed with an ABI Prism 7900HT sequence detection system using the SYBR Green PCR Core Reagent (Applied Biosystems, Foster City, CA) [13].

\section{Results}

\section{Hsp90 is required for protein expression of IKK}

Although it is clear that Hsp90 physically associates with IKK, the role of Hsp90 in IKK expression is still controversial $[7,19]$. To address this discrepancy, we reexamined the effect of GA treatment on IKK expression in numerous cell lines. As shown in Figure 1, GA induced a significant decline of the expression levels of all three IKK subunits in all the cell lines we examined, including 293 cells, HeLa cells, B cells and T cells (also see Figures 2 and 3). The effect of GA was specific, because it had no effect on the expression levels of actin as well as p65 and p100, two members of NF- $\kappa$ B (bottom panels, and data not shown). Thus, it seems that Hsp90 plays a general role in IKK protein expression.

\section{Hsp90 is not required for transcription or translation of IKK}

To explore the mechanism of the GA-mediated decrease of IKK protein levels, we first examined whether GA suppressed IKK mRNA transcription and translation by performing the real-time RT-PCR and polysome fractionation analysis. As a control, the transcription and translation of p100 mRNA was also included. In agreement with the fact that CD40L can activate NF- $\mathrm{KB}$ to induce p100 expression, we found that CD40 antibody treatment dramatically 

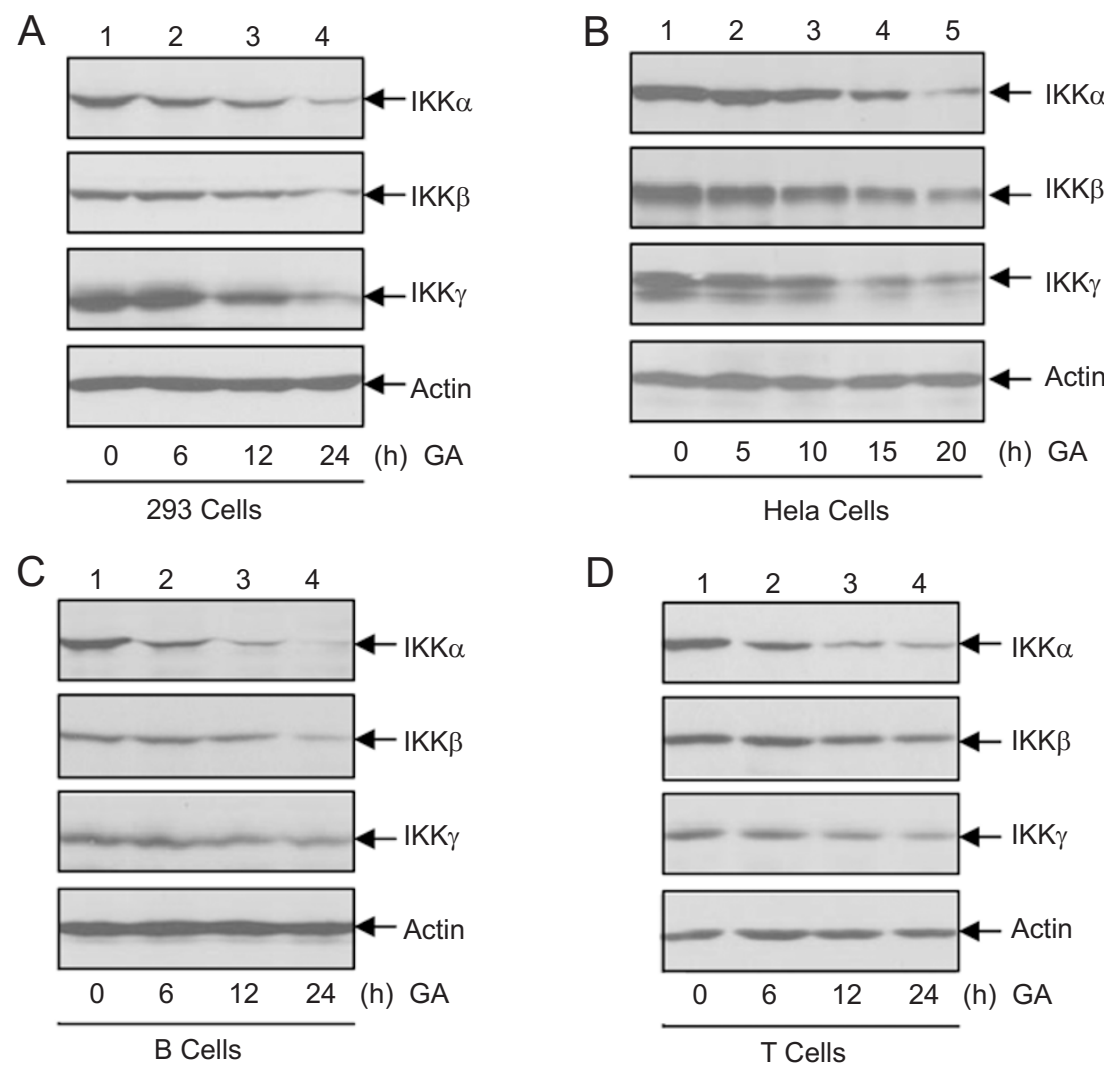

Figure 1 GA induces decrease of the expression levels of IKK proteins in different cells. (A-D) The cells were incubated with GA (10 $\mu \mathrm{M}$ for 293 and HeLa cells; $2 \mu \mathrm{M}$ for B and T cells) for the indicated time periods, followed by IB to examine protein levels of $\mathrm{IKK} \alpha, \mathrm{IKK} \beta, \mathrm{IKK} \gamma$ and actin.

enhanced p100 mRNA transcription and subsequent protein translation (Figure 4, columns 6). On the other hand, GA treatment hardly influenced mRNA transcription and translation of all three IKK subunits (columns 2-4). These data suggested that the decrease of IKK levels is due to GA-induced protein degradation of IKK.

IKK degradation induced by GA is independent of the ubiquitination and proteasome

Since all known Hsp90 clients are degraded by the ubiquitin-proteasome pathway when Hsp90 function is inhibited [4], we investigated the possible role of ubiqutination in GA-mediated degradation of IKK by using ts 20 cells. ts 20 cells are temperature-sensitive (ts) mutant cells expressing a heat-labile ubiquitin-activating enzyme (E1), therefore, defective in the ubiquitin pathway upon heat shock [20]. Surprisingly, heat shock failed to prevent GA-mediated degradation of IKK $\alpha$, IKK $\beta$ or IKK $\gamma$ in these mutant cells (Figure 2A, lane 4). This unexpected result was not due to inefficient inactivation of E1, because heat shock completely blocked the degradation of p53 and IкB $\alpha$ (lane 6).
These results demonstrated that the ubiquitin system is not required for GA-induced IKK degradation.

These results led us to further examine the role of the proteasome in the GA-induced IKK degradation, although the proteasome can mediate either ubiquitin-dependent or -independent proteolysis [21]. To achieve this goal, we inhibited the proteolytic activity of the proteasome using MG132, a specific inhibitor of the proteasome. Consistent with the essential role of the proteasome in the degradation of I $\mathrm{I} \mathrm{B} \alpha$, the direct target of IKK and major inhibitor of NF- $\kappa \mathrm{B}$ [8], addition of MG132 dramatically increased expression level of $\mathrm{I} \kappa \mathrm{B} \alpha$ (Figure 2B, lanes 3 and 5). However, the addition of MG132 failed to increase expression levels of IKKs (lane 5). Most importantly, MG132 could not block GA-induced IKK degradation (lane 3). It is noteworthy that GA treatment alone also significantly enhanced I $\mathrm{B} \alpha$ expression (lane 2), which was correlated perfectly with the IKK degradation induced by GA. Collectively, these studies clearly demonstrated that IKK degradation in the absence of Hsp90 function is independent of both ubiquitination and proteasome, further suggesting that an 


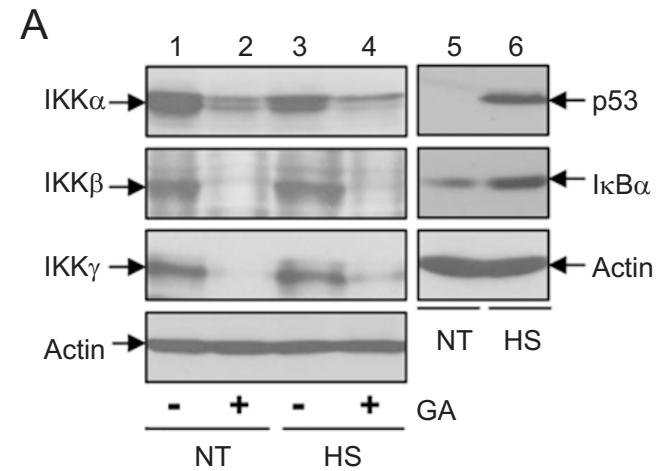

B

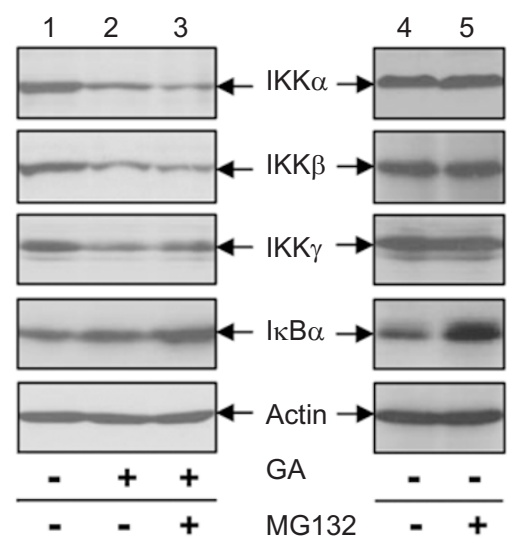

Figure 2 IKK protein degradation induced by GA does not involve the ubiquitin-proteasome system. (A) The ubiquitination system is dispensable for GA-induced IKK degradation. IKK or mock transfected ts 20 cells were incubated at $35{ }^{\circ} \mathrm{C}$ (labeled as NT) or $39^{\circ} \mathrm{C}$ (labeled as HS) in the presence of $10 \mu \mathrm{M}$ GA (+) or DMSO (-) for $20 \mathrm{~h}$, followed by IB to examine protein expression levels of IKKs, actin, p53 and $\mathrm{I} \kappa \mathrm{B} \alpha$. (B) The proteasome is dispensable for GA-induced IKK degradation. 293 cells were incubated with $10 \mu \mathrm{M}$ GA (+) or DMSO (-) in the presence or absence of $25 \mu \mathrm{M}$ MG132 for $20 \mathrm{~h}$, followed by IB to examine expression levels of the indicated proteins.

unidentified mechanism is responsible for the degradation of this Hsp90 client.

\section{GA-mediated IKK degradation is largely mediated by autophagy}

To define the novel mechanism by which IKK is degraded in the absence of Hsp90 function, we examined the possible role of autophagy, because it is the other main system responsible for protein degradation in addition to the proteasome [22-24]. As shown in Figure 3A, AICAR, an inhibitor of autophagy [25], efficiently prevented IKK degradation induced by GA (top three panels, lane 3 ). The role of AICAR is specific, because it failed to protect
Akt, a well-known client of Hsp90, from GA-mediated degradation (middle panel, lane 3). Consistent with previous studies showing that GA-induced degradation of Akt depends on the proteasome [18], inhibition of the proteasome by MG132 rescued Akt from the proteasomal proteolysis (middle panel, lane 4). These studies suggested that autophagy may be responsible for the GA-mediated degradation of IKK.

To validate these biochemical studies, we utilized the Atg5 deficient cells. Atg5 is essential for autophagosome formation, and knockout of Atg5 blocks autophagy [26]. Consistent with the results shown above, knockout of Atg5 also significantly inhibited GA-triggered degradation of IKK (Figure 3B, top three panels, lane 4). In sharp contrast, Atg5 was not required for GA-induced Akt degradation, because Akt was still degraded in the Atg5 null

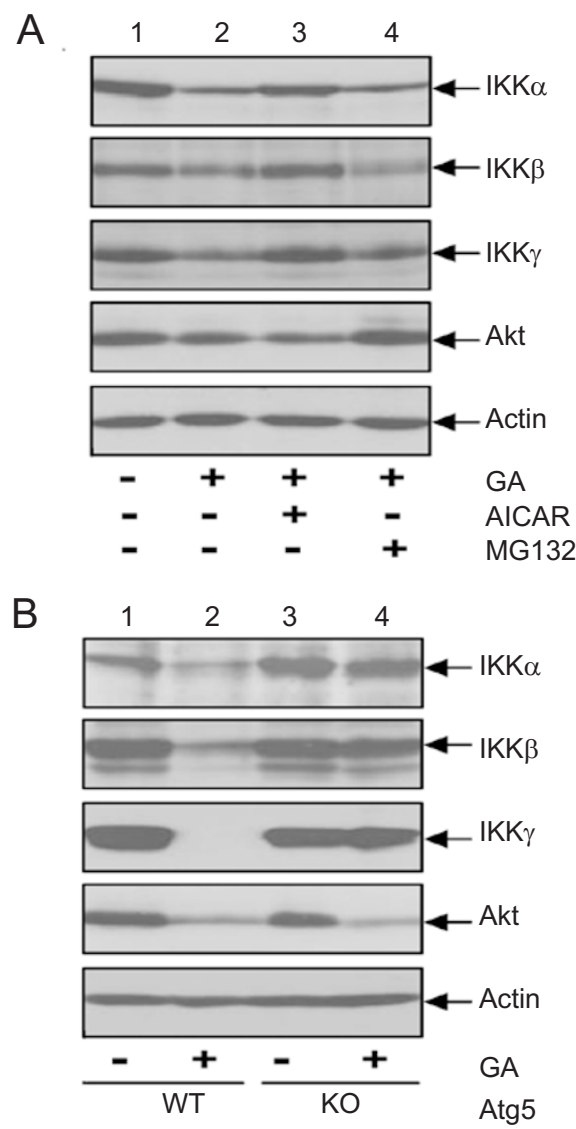

Figure 3 IKK protein degradation induced by GA is largely mediated by autophagy. (A) AICAR, an inhibitor of autophagy, blocks GAinduced IKK degradation. 293 cells were incubated with $10 \mu \mathrm{M}$ GA $(+)$ or DMSO (-) in the presence of AICAR (1 mM), or MG132 (25 $\mu \mathrm{M})$ for $20 \mathrm{~h}$, followed by IB to detect protein levels of IKKs, Akt and actin. (B) Atg5 wild-type or null cells transfected with IKKs or Akt were incubated with $5 \mu \mathrm{M}$ GA (+) or DMSO (-) for $20 \mathrm{~h}$, followed by IB as described in (A). 
A

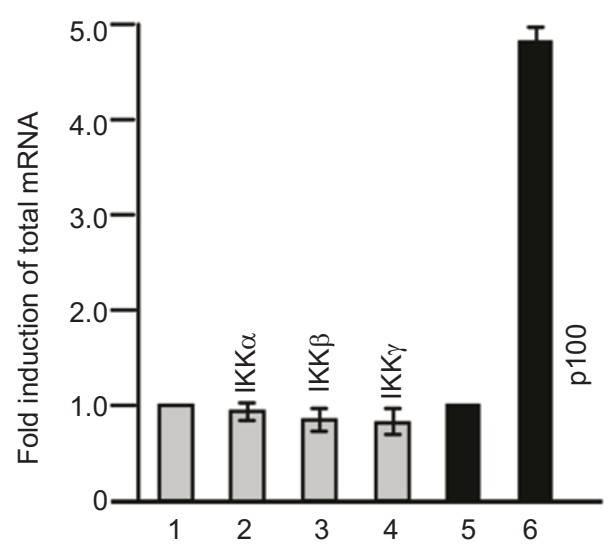

B

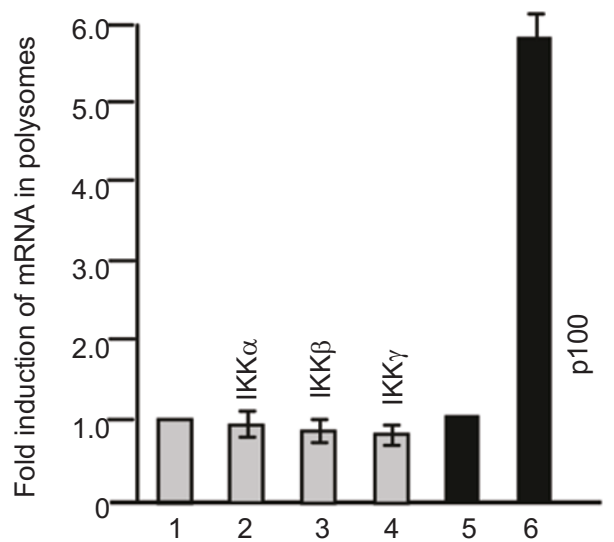

Figure 4 GA treatment does not change RNA transcription or protein translation of IKKs. (A) GA addition has no obvious effect on IKK mRNA transcription. B cells were treated for $10 \mathrm{~h}$ with DMSO (columns 1 and 5), $2 \mu \mathrm{M}$ GA (columns 2-4) or $10 \mu \mathrm{g} / \mathrm{ml}$ anti-CD40 (column 6), followed by RNA extraction and real-time RT-PCR to quantitate mRNAs of IKK $\alpha$, IKK $\beta$, IKK $\gamma$ and $p 100$. The amount of IKK and p100 mRNA was normalized to the level of GAPDH mRNA. The values represented fold change in mRNA abundance relative to the DMSO-treated sample (arbitrarily set as one-fold) and were means + S.E.M. of three independent experiments. (B) GA addition has no obvious effect on IKK protein translation. Polysomes were also isolated from the Ramos B cells in (A), followed by RNA extraction and real-time RT-PCR. The mRNA levels of IKKs and p100 in the polysomes were quantitated as described in (A).

cells (middle panel, lane 4). Thus, IKK degradation in the absence of Hsp90 is largely mediated by autophagy. This is the first evidence that autophagy may function as an alternative mechanism for Hsp90 client degradation when Hsp90 function is absent.

\section{Discussion}

The proteasome and autophagy are two highly conserved

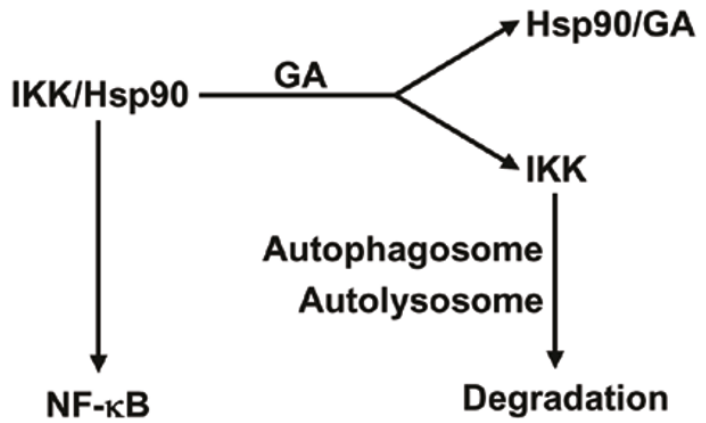

Figure 5 A model of IKK regulation by Hsp90 and autophagy. Under normal conditions, the Hsp90 chaperone binds to nascent IKK proteins (possibly indirectly via co-chaperone $\mathrm{Cdc} 37$ ) and facilitates their maturation, a process essential for IKK stabilization and subsequent $\mathrm{NF}-\kappa \mathrm{B}$ activation. When Hsp90 function is absent (such as inhibition by GA), the nascent proteins of IKK cannot be folded correctly and/or the mature proteins can not maintain the correct conformation, resulting in degradation via the autophagy pathway

mechanisms that are primarily employed for protein degradation within eukaryotes. It is generally believed that autophagy is in principle a nonselective, bulk degradation system of long-lived proteins and organelles; while the proteasome specifically degrades short-lived proteins, including regulatory proteins and misfolded proteins caused by the absence of Hsp90 function. Here, we show that IKK is selectively degraded by autophagy but not by the proteasome when Hsp90 function is inhibited. This study thus provides the first line of evidence showing that autophagy may also selectively degrade proteins and an Hsp90 client can be targeted for degradation via a mechanism distinct from the proteasome.

Consistent with our findings, a recent study clearly demonstrates that catalase is selectively degraded by autophagy upon caspase inhibition. Interestingly, activation of autophagy by nutrient deprival, a prototypic stimulus of autophagy, fails to trigger catalase degradation [27]. Together the emerging evidence strongly indicates that autophagic degradation can be highly selective and regulated, at least under certain situations. Right now, the mechanism by which autophagy specifically selects its cargo is still unknown. One can speculate that the substrate must either be modified before being recognized, or it may contain a specific sequence that can be directly recognized by the autophagy machinery. However, such modification or motif has not yet been identified. Similarly, it is unknown which autophagy gene products are involved in targeting specific proteins. The other possibility is that certain protein or protein complex is responsible for the selection. For example, the chaperone protein complex containing Hsp90 and Hsc70 has been found to recognize and deliver 
specific proteins directly to lysosome for degradation, a process called CMA [28]. However, it is obvious that the IKK degradation induced by GA is not mediated by CMA, because the GA treatment actually disrupts the association between IKK and Hsp90 [7, 19]. More importantly, the IKK degradation requires Atg5 (Figure 3), a key player in autophagosome formation [26]. On the other hand, CMA does not require ATG5/autophagosome and vesicular traffic [6]. Given the significance of autophagy-mediated IKK degradation, it will be of great interest to determine the molecular basis of selective action of autophagy.

Autophagy and Hsp90 both contribute to cell survival under stress conditions. Paradoxically, they function oppositely at the molecular level [3, 4, 22-24, 29, 30]. For example, autophagy degrades protein, while Hsp90 is required for stabilization of many proteins by assisting the folding and maintenance of the newly translated proteins. Additionally, autophagy suppresses tumor development, whereas Hsp90 facilitates tumor progression and renders tumor cells resistant to a variety of apoptosis-inducing stimuli such as radiation. The data in the present study establish a molecular link between autophagy and $\mathrm{Hsp} 90$ and demonstrate that $\mathrm{IKK} / \mathrm{NF}-\kappa \mathrm{B}$ serves as the nexus in between. In this novel signaling pathway, Hsp90 positively regulates IKK stability and NF- $\mathrm{KB}$ activation. When Hsp90 function is lost (such as inhibition by GA), IKK proteins cannot be folded correctly and are accordingly degraded by autophagy, resulting in $\mathrm{NF}-\kappa \mathrm{B}$ inactivation (Figure 5). Given a causative role of $\mathrm{NF}-\kappa \mathrm{B}$ in tumorigenesis and resistance of malignant cells to apoptosis-based tumor surveillance $[8,9]$, our data here thus may provide an important insight into the molecular mechanism of the tumor suppression function of autophagy and the anti-tumor activity of GA. As GA has been used in clinic trials for metastatic cancers, the rationale may also be applicable to all the NF- $\mathrm{KB}$ associated diseases such as various tumors and autoimmune diseases.

\section{Acknowledgments}

We thank Drs Covey L for the Ramos cells, Mizushima $\mathrm{N}$ for Atg5 null MEFs and Ozer HL for ts 20 cells. This study was assisted partially by research grants from the New Jersey Commission on Cancer Research, Fifth District AHEPA Cancer Research Foundation, American Cancer Society RSG MGO-110116 and National Institutions of Health 1R01 CA116616 to GX.

\section{References}

1 Levine B, Klionsky DJ. Development by self-digestion molecular mechanisms and biological functions of autophagy. Dev Cell 2004; 6:463-477.

2 Lodish H, Berk A, Matsudaira P, et al. Molecular Cell Biology. 5th Edition, New York: WH Freeman and Company. 2004: 59.

3 Dai C, Whitesell L. HSP90: a rising star on the horizon of anticancer targets. Future Oncol 2005; 1:529-540.

4 Zhang H, Burrows F. Targeting multiple signal transduction pathways through inhibition of Hsp90. J Mol Med 2004; 82:488499.

5 Whitesell L, Mimnaugh EG, De Costa B, Myers CE, Neckers LM. Inhibition of heat shock protein HSP90-pp60v-src heteroprotein complex formation by benzoquinone ansamycins: essential role for stress proteins in oncogenic transformation. Proc Natl Acad Sc USA 1994; 91:8324-8328.

6 Majeski AE, Dice JF. Mechanisms of chaperone-mediated autophagy. Int J Biochem Cell 2004; 36:2435-2444.

7 Chen G, Cao P, Goeddel DV. TNF-induced recruitment and activation of the IKK complex require Cdc37 and Hsp90. Mol Cell 2002; 9:401-410.

8 Xiao G, Rabson A, Young W, Qing G, Qu Z. Alternative pathways of NF-kappaB activation: a double-edged sword in health and disease. Cytokine Growth Factor Rev 2006; 17:281-293.

9 Karin M. Nuclear factor-kappaB in cancer development and progression. Nature 2006; 441:431-436.

10 Xiao G, Harhaj EW, Sun SC. Domain-specific interaction with the I kappa B kinase (IKK) regulatory subunit IKK gamma is an essential step in tax-mediated activation of IKK. J Biol Chem 2000; 275:34060-34067.

11 Qing G, Qu Z, Xiao G. Regulation of NF-kappaB2 p100 processing by its cis-acting domain. J Biol Chem 2004; 280:18-27.

12 Harhaj EW, Good L, Xiao G, et al. Somatic mutagenesis studies of NF-kappaB signaling in human T cells: evidence for an essential role of IKK gamma in NF-kappaB activation by T-cell costimulatory signals and HTLV-I Tax protein. Oncogene 2000; 19:1448-1456.

13 Qing G, Qu Z, Xiao G. Stabilization of basally translated NFkappaB-inducing kinase (NIK) protein functions as a molecular switch of processing of NF-kappaB2 p100. J Biol Chem 2005; 280:40578-40582.

14 Qu Z, Qing G, Rabson A, Xiao G. Tax deregulation of NFkappaB2 p100 processing involves both beta-TrCP-dependent and -independent mechanisms. J Biol Chem 2004; 279:4456344572.

15 Xiao G, Harhaj EW, Sun SC. NF-kappaB-inducing kinase regulates the processing of NF-kappaB2 p100. Mol Cell 2001; 8:401-409.

16 Xiao G, Civijic ME, Fong A, et al. Retroviral oncoprotein Tax induces processing of NF-kappaB2/p100 in T cells: evidence for the involvement of IKKalpha. EMBO J 2001; 20:6805-6815.

17 Qing G, Xiao G. Essential role of IkappaB kinase alpha in the constitutive processing of NF-kappaB2 p100. J Biol Chem 2005; 280:9765-9768.

18 Basso AD, Solit DB, Chiosis G, et al. Akt forms an intracellular complex with heat shock protein 90 (Hsp90) and Cdc37 and is destabilized by inhibitors of Hsp90 function. J Biol Chem 2002; 277:39858-39866.

19 Broemer M, Krappmann D, Scheidereit C. Requirement of Hsp90 activity for IkappaB kinase (IKK) biosynthesis and for constitutive and inducible IKK and NF-kappaB activation. Oncogene 2004; 23:5378-5386.

20 Chowdary DR, Dermody JJ, Jha KK, Ozer HL. Accumulation of p53 in a mutant cell line defective in the ubiquitin pathway. Mol Cell Biol 1994; 14:1997-2003. 
21 Orlowski M, Wilk S. Ubiquitin-independent proteolytic functions of the proteasome. Arch Biochem Biophys 2003; 415:1-5.

22 Edinger AL, Thompson CB. Defective autophagy leads to cancer. Cancer Cell 2003; 4:442-424.

23 Mizushima N. The pleiotropic role of autophagy: from protein metabolism to bactericide. Cell Death Differ 2005; 12 Suppl 2:1535-1541.

24 Shintani T, Klionsky DJ. Autophagy in health and disease: a double-edged sword. Science 2004; 306:990-995.

25 Samari HR, Seglen PO. Inhibition of hepatocytic autophagy by adenosine, aminoimidazole-4-carboxamide riboside, and N6-mercaptopurine riboside. Evidence for involvement of ampactivated protein kinase. J Biol Chem 1998; 273:23758-23763.

26 Kuma A, Hatano M, Matsui M, et al. The role of autophagy dur- ing the early neonatal starvation period. Nature $2004 ; \mathbf{4 3 2}$ : 1032 1036.

27 Yu L, Wan F, Dutta S, et al. Autophagic programmed cell death by selective catalase degradation. Proc Natl Acad Sci USA 2006; 103:4952-4957.

28 Agarraberes FA, Dice JF. A molecular chaperone complex at the lysosomal membrane is required for protein translocation. J Cell Sci 2001; 114:2491-2499.

29 Kondo Y, Kanzawa T, Sawaya R, Kondo S. The role of autophagy in cancer development and response to therapy. Nat Rev Cancer 2005; 5:726-734

30 Levine B, Yuan J. Autophagy in cell death: an innocent convict? J Clin Invest 2005; 115:2679-2688. 